\title{
HIGH OR LOW FREQUENCY TENS IN PATIENTS WITH KNEE OSTEOARTHRITIS- WHAT WORKS BETTER?
}

\author{
Mukesh Yadav ${ }^{* 1}$, Pooja Attrey ${ }^{2}$, Shalu Kamal ${ }^{3}$. \\ ${ }^{* 1,2}$ Assistant Professor, T.D.T.R.D.A.V. Institute of Physiotherapy \& Rehabilitation, Professor Colony, \\ Yamunanagar, Haryana, India. \\ ${ }^{3}$ Working as clinical physiotherapist, Ambala, Haryana, India.
}

\section{ABSTRACT}

Background: TENS is a commonly used non-pharmacological intervention for the management of pain. However, what role does it play on the pain as well as other parameters is still in doubt.

Objective: To compare the effects of high and low frequency TENS on patients with Knee Osteoarthritis.

Materials and Methods: Study Designed as a an Experimental design, and data were collected form various Private hospitals, Yamunanagar among them 45 Diagnosed patient of Knee OA fulfilled the inclusion criteria were taken for the study and randomly assigned in three groups: Group 1 received HF TENS, Group 2 received LF TENS, Group 3 received Placebo TENS along with conventional strengthening physiotherapy protocol. The treatment was given for 2 weeks. Outcome measures of PPT, TUG, WOMAC and NPRS were evaluated before the starting of treatment protocol (day 0), at end of first week and then at the end of treatment i.e., day 0, 7 and 14.

Results: There was significant decrease in pain at rest with HF TENS, LF TENS and placebo TENS. Both HF and LF TENS produced significant improvement in PPT and functional abilities as seen by WOMAC and TUG as compared to Placebo group. However there was no significant difference between the effect of HF and LF TENS on patients with knee OA.

Conclusion: There was no significant differences between the effects of High and Low frequency TENS in patients with Knee OA. Both could be used in management of pain at rest and during functional tasks in patients with knee OA

KEY WORDS: Osteoarthritis, TENS, TUG, WOMAC, PPT, NPRS.

Address for correspondence: Dr. Mukesh Yadav. MPT, Assistant Professor, T.D.T.R.D.A.V. Institute of Physiotherapy \& Rehabilitation, Professor Colony, Yamunanagar - 135 001, Haryana, India.

E-Mail:mukesh.mpt@gmail.com; poojaattrey6@gmail.com

\section{Access this Article online}

Quick Response code

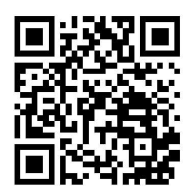

DOI: $10.16965 /$ ijpr.2017.172
International Journal of Physiotherapy and Research

ISSN 2321- 1822

www.ijmhr.org/ijpr.html

Received: 04-05-2017

Peer Review: 06-05-2017

Accepted: 05-06-2017

Revised: None
Published (0): 20-07-2017

Published (P): 11-08-2017

\section{BACKGROUND}

Osteoarthritis is the most common joint disorder with the prevalence that increases with age [1]. In age of $14-15$ years only $3 \%$ males and $2 \%$ of females are found to be affected and in age group of $45-54$ years $25 \%$ of male population and $30 \%$ of female population suffered from OA and over the age of $65 \%$ of males and $68 \%$ of females are affected. Osteoarthritis of knee is the third in frequency behind that of spine, hip and has greater cost and more associated disability than OA of any other joint. Therefore Lawrence reveals that OA of knee was the second ranking (after cardiovascular disease) of permanent incapacity in people over 50 years of age [2].

Osteoarthritis is a chronic degenerative disorder primarily affecting the articular cartilage of 
synovial joints, with eventual bone remodeling and overgrowth of margins of joints. It is characterized by pain, swelling, stiffness for less than 30 minutes, crepitus, bony enlargement, limitation of range of motion, instability and tenderness. The common physical impairments associated with knee $\mathrm{OA}$ are pain, decreased range of motion and quadriceps muscle weakness an addition to the episodes of knee instability [3]. In addition to traditional physiotherapy, such as, physical training, TENS is commonly used by physiotherapists for pain relief in these patients [4]. TENS is an inexpensive, non-invasive intervention used to manage a wide variety of painful conditions. It is often used as a non- pharmacological option for alleviating chronic and post- operative pain [5]. TENS involves the application of electrical current to the skin and can be set to different frequencies, durations and intensities. This results in a large recruitment of sensory nerve fibers and mechanoreceptors. Previous studies showed that TENS increases pressure pain thresholds in people who are healthy and reduces mechanical and heat hyperalgesia in arthritic animals $[6,7]$.

Dosing is critical to the effectiveness of TENS. ${ }^{7}$ Animal studies show that both high-frequency TENS (HF-TENS, $>50 \mathrm{~Hz}$ ) and low-frequency TENS (LF-TENS, $<10 \mathrm{~Hz}$ ), delivered at an intensity of $90 \%$ of the motor threshold (strong sensory intensity), reduced pain sensitivity in arthritic animals. Several studies of HF-TENS in people, who are healthy, show that higher intensities, generally described as strong but comfortable, resulted in greater pain reduction [8].

Previous studies have examined the effectiveness of TENS in managing knee pain. Stimulation duration is a parameter which could influence the duration of post stimulation analgesia [9]. However, recent research studies have shown that analgesic effects of endogenous opioids takes time and it can last for sometime before they decay. The accumulation or depletion of endogenous opioids can influence, the amplitude and duration of post stimulation analgesia of TENS [10]. For many years, the research on TENS have been carried out and the ranges 2 and $100 \mathrm{~Hz}$ were the mostly used effective frequencies for the management of $O A$ knees [11].
Aims \& objectives: To find out if there is any significant difference in the effectiveness of high and low TENS in improving the pain(NPRS), pain sensitivity(PPT), mobility (TUG) and functional disability (WOMAC) in patients with knee Osteoarthritis.

Objective of this study is to compare the effect of high TENS $(100 \mathrm{~Hz})$ and low TENS $(4 \mathrm{~Hz})$ in patients with knee Osteoarthritis.

\section{MATERIALS AND METHODS}

Study has conducted in Private hospitals, Yamunanagar, with 45 subjects and experiment has designed as an experimental design with randomized sampling.

Inclusion Criteria: Age between 50 to 80 years, Unilateral Knee OA, Symptomatic primary OA for at least 3 months, Fulfillment of Altman criteria of idiopathic $O A$, Tenderness on the medial joint line.

Exclusion Criteria: Diagnosed cases of diabetes, neurological disorders' malignancy, Rheumatoid arthritis, Knee surgery in last 6 months, Patients who are involved in exercise programs as well as PT programs, Open wounds and defective skin sensations around the knee, Any other knee pathology like ruptured ligament or fracture.

Instrumentation used were TENS machine, Pressure algometer, Inch Tape, Stop watch.

Procedure: On the basis of the inclusion criteria,48 subjects were selected after explaining the procedure to them and taking their consent, 45 subjects( 3 dropped out) were randomly assigned to three groups: HF TENS group, LF TENS group and placebo TENS group.

Group 1: High frequency TENS ( freq. $=100$ Hz.), Pulse width $=50$ microseconds, Duration: $40 \mathrm{~min}$, Waveform = Rectangular, Strong \& comfortable intensity.

Group 2: Low frequency TENS ( freq. $=4 \mathrm{~Hz}$ ), Pulse width $=200$ microseconds, duration: 40 min, Waveform = Rectangular, Strong \& comfortable intensity.

Group 3: Placebo TENS group(control group), Duration: $40 \mathrm{~min}$, Intensity at zero, Conventional physiotherapy treatment (strengthening exercises) were given to each group. 
Subjects were instructed not to take part in any other treatment program.

TENS Application: Position of patient: Patient in supine lying, 2 channels were used with electrodes placed in the cross manner. Parameters were recorded at day 0 , day 7 and day 14

Pressure algometer: The subject was instructed to lie supine on a couch and were told that when pressing on the tender point with even a minimal pressure it will cause them pain. Starting from neutral (gauge at 0), the pressure was exerted on the tender point and the pointer then turned to the reading at which the patient feels pain .Three readings were taken at the medial joint line marked $1 \mathrm{~cm}$ apart. Mean was calculated in kgs. Then gauge was reset to zero before taking any reading.

Timed Up and Go Test: Instructions given to the patient to get up from the chair and walk approx $9.8 \mathrm{ft}$., take a turn and then come back to chair. Three readings of time taken were noted and mean was calculated in seconds.

WOMAC: It is a self reported questionnaire for patient. It is composed of 24 items worth 96 total score measuring pain, stiffness and functional limitation. Individual score for each subject were taken.

NPRS: The line of $10 \mathrm{~cm}$ was drawn on the paper and patient was asked to rate his pain in between 0 to 10 .

Data Analysis: Data analysis was done by SPSS 21 software. $p<=0.05$ is considered significant. Mean and standard deviation of all the four parameters taken on day 0 , day 7 and day 14 were calculated. Statistical analysis was done by using $T$ test.

\section{RESULT}

A total of 45 subjects with a mean age group of 60.82 included in the research study. Between groups analysis is done by independent $T$ test. There is no significant improvement in NPRS,WOMAC, TUG and also in PPT from day 0 to day 14.

Within group analysis is done by using paired $T$ test. There is a significant improvement in all the three groups from day 0 to day 14 in NPRS, While a significant improvement in WOMAC, TUG and PPT in group 1 \&2. However there is no significant improvement in control group.

Table 1: Basic Characteristics.

\begin{tabular}{|c|c|c|c|}
\hline Groups & Age in years & No. of M \& F & $\begin{array}{c}\text { Knee pain duration } \\
\text { in months }\end{array}$ \\
\hline Group 1 & $57.13 \pm 6.51$ & $10 \mathrm{M} \& 5 \mathrm{~F}$ & $1.57 \pm 1.65$ \\
\hline Group 2 & $63.93 \pm 10.47$ & $9 \mathrm{M} \& 6 \mathrm{~F}$ & $2.76 \pm 2.45$ \\
\hline Group 3 & $61.4 \pm 8.59$ & $7 \mathrm{M} \& 8 \mathrm{~F}$ & $2.46 \pm 2.36$ \\
\hline
\end{tabular}

Table 2: Percentage of improvement in the variables.

\begin{tabular}{|c|c|c|c|}
\hline Parameters & Group 1 & Group 2 & Group 3 \\
\hline NPRS & 48.52 & 50.05 & 51.1 \\
\hline WOMAC & 46.5 & 51.5 & 2.12 \\
\hline TUG & 29.1 & 25.2 & 1.61 \\
\hline
\end{tabular}

Graph 1: Comparison of NPRS of all the three groups.

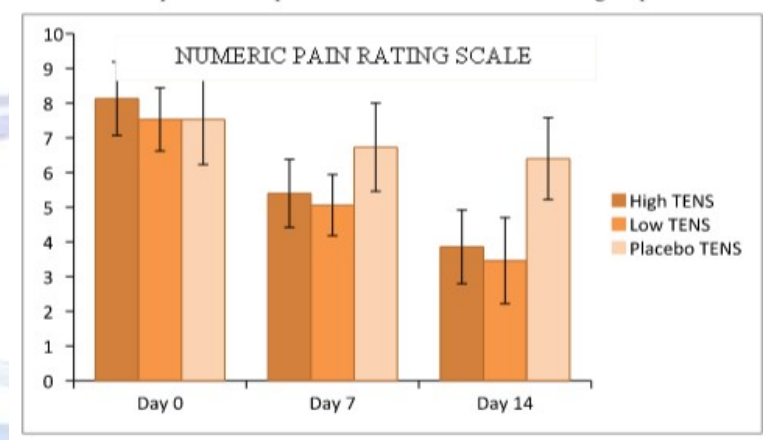

Graph 2: Comparison of WOMAC of all the three groups.

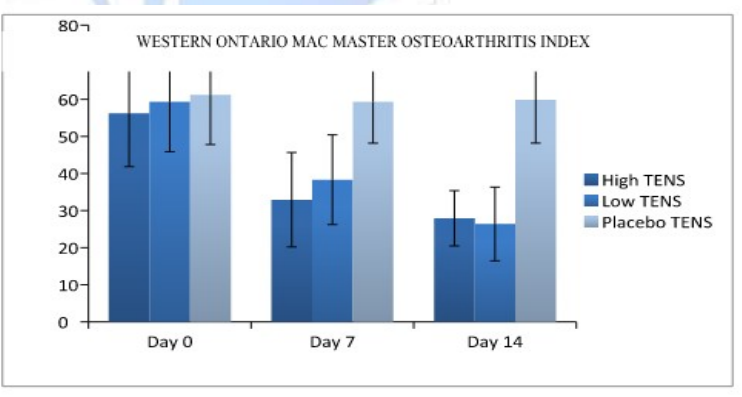

Graph 3: Comparison of TUG of all the three groups.

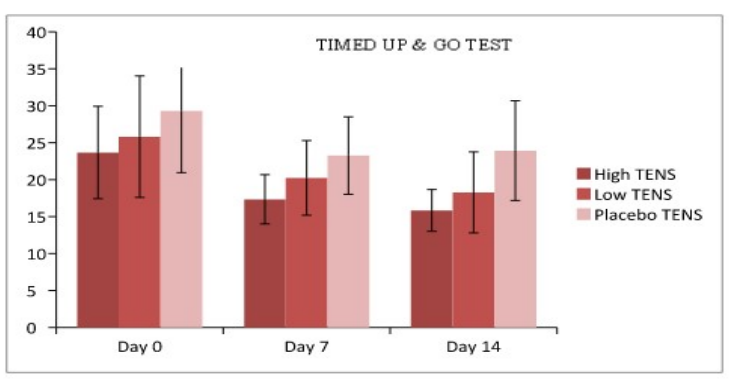

Graph 4: Comparison of Pressure Pain Threshold.

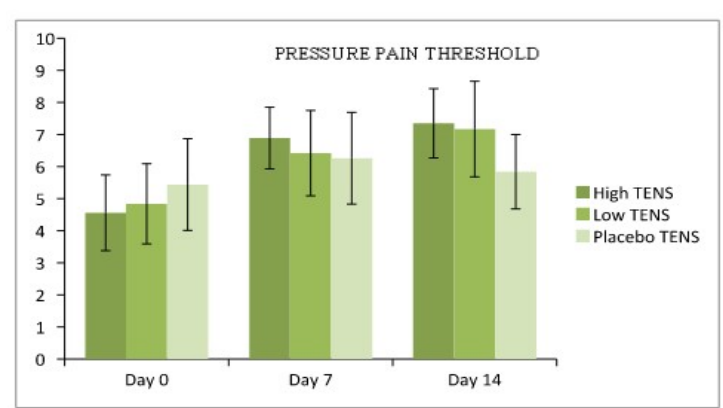




\section{DISCUSSION}

The main objective of the present study is to compare between the effects of High and Low frequency TENS in patients with Knee Osteoarthritis. The results have shown that there is no significant difference in the effects of high and low TENS between the groups on any of the variables.

Effect of TENS on Pressure pain threshold: Present study shows that both High TENS and Low TENS cause a reduction in primary hyperalgesia seen in osteoarthritis patients, as the pressure pain threshold found to increase over the tender points at medial joint line. TENS reduces excitability of nociceptor neurons in the CNS and higher intensities produced greater reduction in excitability therefore the changes in PPT with TENS are likely to be mediated by reduced central neuron excitability [12].

Effect of TENS on Pain and function: All four variables (Pain, function, TUG \& PPT) have shown significant improvement within the group. Both High and Low TENS lead to decrease in pain and significant improvement in function seen by WOMAC \& TUG over a period of 14 days The effect of placebo $\&$ active TENS is same on pain at rest as seen by NPRS because all 3 groups produce a significant result after 14 days [12].

The reduction in pain by TENS can be explained by Pain gate mechanism. High TENS stimulate A beta fibers, not because of high frequency but because of the short pulse width (around 50 microseconds) that results in the preferential recruitment of large diameter nerve fibers [13]. They are usually delivered between the 100 and 200 pps to produce a strong comfortable and non painful par aesthesia. It produces segmental analgesia with rapid onset in less than 30 minutes after the machine is switch on and rapid offset with less than 30 minutes after the machine is switch off. The main mechanism of analgesic action is presynaptic inhibition. It means that activity generated in A beta fibers inhibits ongoing activity in dorsal horn of the spinal cord [14].

Low TENS uses longer pulse duration more than 300 microseconds and activate A delta fibres to produce the inhibition of ascending transmision of $\mathrm{C}$ fibers signals and activation of descending pathways resulting in endorphins \& encephalin release [13]. They are usually delivered approx 100 pps within the burst. The main mechanism of analgesic action is extrasegmental and segmental by postsynaptic inhibition. It produces extra segmental and segmental analgesia with delayed onset of greater than $\mathbf{3 0}$ minutes after switch on and delayed offset of greater than 1 hour after switch off. There is an activation of structures in the extra segmental mechanism which form the descending inhibitory pathways such as Nucleus Raphe Magnus, Nucleus Raphe Gigantocellularis and Periaqueductal Grey matter(PAG). The main desired outcome is strong comfortable phasic muscle contraction. Phasic muscle contraction produced during low TENS generates activity in small diameter muscle afferents leading to the activation of descending inhibitory pathways [14].

Effect of Intensity of TENS: Cochrane Review done in 2009 supported the use of high intensity TENS in people with knee OA. Present study TENS was applied at strong and comfortable intensities. The intensity of stimulation has been positively correlated with the change in pressure pain threshold produced by TENS application. In this study the intensity was adjusted over time as is common in clinical practice. Adjusting the TENS intensity during the treatment prevents accommodation of sensory nerve fibers to TENS currents [12]. The total current applied with HFTENS is greater than that applied with LFTENS. When the same intensity and pulse duration are used [13].

Earlier studies have shown that Low frequency TENS has strong intensity produced effects equivalent those obtained with high frequency TENS at a strong intensity. Opioid mediated analgesia occurs at sensory intensities with both high and low frequency TENS. Thus the present study delivered a strong intensity and studied the effect of frequency on the patients [12].

This study delivered TENS for $\mathbf{4 0}$ minutes treatment , also supported by Gladys L.Y. Cheing et al in 2003, concluding 40 minutes treatment duration of TENS is optimal for the management of Osteoarthritis knee pain.

The gradual onset of TENS analgesia could be 
explained by the time lag releasing the endogenous opioids similarly the gradual offset could be due to prolonged defect of the endogenous opioids substances before decaying [15].

Active TENS response has shown more superior results when compared to placebo supported by Karen Grimmer [13].

Both NMES and TENS were proved very effective modality in reducing the knee pain and in improving functional quality of life in research done by Shivkumar et .al on OA knee subjects [15]. The functional Improvement can be explained by the fact that due to increase pain threshold and decreased musculoskeletal pain allows the subject to effectively perform various muscular activities and joint range of motion that gradually improves the functional ability of the subject.

As found in the study that functional improvement (TUG \&WOMAC) is significant within the placebo group, indicates the effect of conventional strengthening exercises given. This has been supported by Lee et.al who concluded that strengthening exercises regime can be considered as an adjunct to treatment and better rehab strategy in reducing the knee pain [17].

Clinical implications: The use of various outcome measures, different frequencies, high intensity and log duration provides insight for the better management of Knee OA pain with TENS.

Limitations of the study: Small sample size and duration of the treatment is shorter.

Future scope: The effect of Brief Intense TENS could be studied. The parameters of TENS for patients with severe pain and less severe pain should be differentially studied.

\section{CONCLUSION}

The study concluded that there is no significant difference in the effects of high and low TENS between the groups on any of the variables (NPRS,TUG,WOMAC and PPT) .Both could be used in the management of pain at rest and during functional tasks in patients with Knee OA.

\section{ABBREVIATIONS}

NPRS - Numeric pain rating scale

TUG - Timed up and go test

PPT - Pressure pain tolerance
WOMAC - Western Ontario and Mc Master University Osteoarthritis Index

HFTENS - High frequency TENS

LFTENS- Low Frequency TENS

TENS - Transcutaneous Electrical Nerve Stimulation

\section{Conflicts of interest: None}

\section{REFERENCES}

[1]. Vansaase, Van Romunde et al. Epidemiology of osteoarthritis comparison of radiological osteoarthritis in Dutch population with that in Jo other population.Ann Rheumatology. 1989;48:271.

[2]. Edstron G . Epidemiology : Rheumatism in populations $2^{\text {nd }}$ Edition.

[3]. Lnkhorst GJ et al. The relationship of functional capacity, pain and isometric and isokinetic torques in osteoarthritis of knee. Scand Journal of Rehabilitation Med. 1985;17:167-72.

[4]. Martin DF. Pathomechanics of Knee Osteoarthritis. Med sports and exercises 1994;14229-1434.

[5]. Crooke TDV et.al features of OA in elderly.Clin.rheumatol. dis 1986:155-172.

[6]. Cheing GLY,Hui Chan: The motor dysfunction of patients with OA knee in Chinse populations Arthritis Care Resp 2001,42:12-68

[7]. Philadelphia panel,evidence based clinical practice guidelineson selected Rehab interventions:Phys ther. 2001,42:12-68

[8]. Walsh, D., The evolution of TENS. Hong Kong Physiotherapy Journa, 2003. 21: p. 1-4.

[9]. CG Vance et.al: Effects of TENS on Pain, Pain sensitivity and function in knee Osteoarthritis. Phys.Ther.Journal.july 2012:92(7):898-910

[10]. G.L.Chieng, Pearl Law. Optimal stimulation frequency for the management of Knee Osteoarthritis J.Rehab Med.2004;36:220-225.

[11]. E.R. Draper et.al. Improvement in function after the valgus bracing of knee. British editorial Society of bone and joint surgery. 2000;82-B(7):1001-1004.

[12].Electrotherapy Explained, Fourth Edition, Val Robertson, Alex Ward John Low and Ann Read.

[13]. Ma YT, Sluka KA. Reduction in inflammation-induced sensitization of dorsal horn neurons by transcutaneous electrical nerve stimulation in anesthetized rats. Exp Brain Res. 2001;137:94-102.

[14]. Karen Grimmer A controlled double blind study comparing the effects of strong burst mode and high rate TENS on painful osteoarthritic knees Aust. J.Physiother 1992;38;49-56.

[15]. H.B.Shivkumara et al. A Comparative Study between the efficacy of NMES and Quadriceps Isometrics Exercises versus TENS and Quadriceps Isometrics Exercises in Patients Suffering From Acute Knee OA. Journal of Evaluation of Medical and Dental Sciences 2014;3(17):4516-4524. 
[16]. Law PP, Cheing GL, Tsui AY. Does transcutaneous electrical nerve stimulation improve the physical performance of people with knee osteoarthritis?. JCR: Journal of Clinical Rheumatology. 2004 Dec 1;10(6):295-9.

[17]. Cheing GL, Tsui AY, Lo SK, Hui-Chan CW. Optimal stimulation duration of tens in the management of osteoarthritic knee pain. Journal of rehabilitation medicine. 2003 Mar 1;35(2):62-8.
[18]. Gladys Chieng, Pearl.L.W.Law,Optimal Stimulation frequency for the management of Osteoarthritis in KneesOsteoarthritis;J.Rehab Med.2004;36 220-22.

[19]. Mascarin NC, Vancini RL, dos Santos Andrade M, de Paiva Magalhães E, de Lira CA, Coimbra IB. Effects of kinesiotherapy, ultrasound and electrotherapy in management of bilateral knee osteoarthritis: prospective clinical trial. BMC musculoskeletal disorders. 2012 Sep 22;13(1):182.

How to cite this article:

Mukesh Yadav, Pooja Attrey, Shalu Kamal. HIGH OR LOW FREQUENCY TENS IN PATIENTS WITH KNEE OSTEOARTHRITIS-WHAT WORKS BETTER? Int J Physiother Res 2017;5(4):2203-2208. DOI: 10.16965/ijpr.2017.172 